\title{
Action boundary proximity effects on perceptual-motor judgements
}

Caleb D. Johnson, $\mathrm{PhD}^{\mathrm{a}, 1}$, Alice D. LaGoy, $\mathrm{MS}^{\mathrm{a}}$, Gert-Jan Pepping, $\mathrm{PhD}^{\mathrm{b}}$, Shawn R. Eagle, $\mathrm{PhD}^{\mathrm{a}}$, Anne Z. Beethe, $\mathrm{MA}^{\mathrm{a}}$, Joanne L. Bower, $\mathrm{PhD}^{\mathrm{c}}$, Candice A. Alfano, $\mathrm{PhD}^{\mathrm{d}}$, Richard J. Simpson, $\mathrm{PhD}^{\mathrm{e}}$, Christopher Connaboy, $\mathrm{PhD}^{\mathrm{a}}$

a. Neuromuscular Research Laboratory, Department of Sports Medicine and Nutrition, University of Pittsburgh, 3860 S. Water St., Pittsburgh, PA, 15203

b. School of Exercise Science, Australian Catholic University, Brisbane Campus, 110 Nudgee Rd., Banyo QLD 4014, Australia

c. Department of Psychology, De Montfort University, The Newarke, Leicester, UK

d. Department of Psychology, University of Houston, 3695 Cullen Boulevard-Rm 126, University of Houston, Houston, TX 77204-5022

e. Department of Nutritional Sciences, The University of Arizona, 1177 E. Fourth Street, Shantz Building, Tucson, AZ 85721

Short Title: Effects of action boundary

\author{
Abstract Word Count: 250 \\ Manuscript Word Count: 3,611 \\ References: 25 \\ Tables: 4
}

Figures: 3

\section{Corresponding Author:}

Christopher Connaboy

3860 S. Water St., Pittsburgh, PA, 15217

connaboy@pitt.edu

Phone: 412-246-0460

Fax: 412-246-0461 


\section{ABSTRACT:}

INTRODUCTION: Designed as a more ecological measure of reaction times, the PerceptionAction Coupling Task (PACT) has shown good reliability and within-subject stability. However, a lengthy testing period was required. Perceptual-motor judgements are known to be affected by proximity of the stimulus to the action boundary. The current study sought to determine the effects of action boundary proximity on PACT performance, and whether redundant levels of stimuli, eliciting similar responses, can be eliminated to shorten the PACT. METHODS: 9 men and 7 women completed 4 testing sessions, consisting of 3 familiarization cycles and 6 testing cycles of the PACT. For the PACT, subjects made judgements on whether a series of balls presented on a tablet afford "posting" (can fit) through a series of apertures. Eight ratios of ball to aperture size (B-AR) were presented, ranging from 0.2 to 1.8 , with each ratio appearing 12 times (12 trials) per cycle. Reaction times and judgement accuracy were calculated, averaged across all B-ARs. Ratios and individual trials within each B-AR were systematically eliminated. Variables were re-averaged, and intraclass correlation coefficients (ICC) and coefficients of variation $\left(\mathrm{CV}_{\mathrm{TE}}\right)$ were calculated in an iterative manner. RESULTS: With elimination of the 0.2 and 1.8 B-ARs, the PACT showed good reliability ( $\mathrm{ICC}=0.81-0.99)$ and consistent withinsubject stability $\left(\mathrm{CV}_{\mathrm{TE}}=2.2-14.7 \%\right)$. Reliability $(\mathrm{ICC}=0.81-0.97)$ and stability $\left(\mathrm{CV}_{\mathrm{TE}}=2.6-\right.$ $15.6 \%$ ) were unaffected with elimination of up to 8 trials from each B-AR. DISCUSSION: The shortened PACT resulted in an almost $50 \%$ reduction in total familiarization/testing time required, significantly increasing usability.

KEYWORDS: PACT, Perception-action Coupling Task, affordance, reliability, reaction time 


\section{INTRODUCTION:}

An action boundary can be defined as a critical point that limits a particular action. That is, an action boundary is the point where an individual's capabilities are exceeded by the movement demands and the actor must adjust their movement strategy for the resulting action to be successful. ${ }^{10}$ Action boundaries are derived from Gibson's theory of affordances, or the possibilities for action within a given environment. ${ }^{11}$ For example, the action boundary for an individual deciding whether they can step up on a raised surface would be the maximum surface height for which they could successfully push themselves up to using their legs. If they encountered a surface that was above this height, they would be forced to select another movement strategy for successful action, such as pushing themselves up on the surface with their arms.

The ability of an individual to accurately judge their action boundaries and alter their behavior accordingly reflects their accuracy of perceptual-motor control and has broad implications for the successful control of movement, agency and decision-making. ${ }^{24}$ Inadequate attunement to these boundaries, defined as the process of calibrating an individual to their action boundaries, has been shown to result in altered postural control and movement patterns, increased latency in reaction time measures, and overall decreases in task performance. ${ }^{8,17,18}$ Summarily, the level to which someone is accurately attuned to their action boundaries likely holds significant implications for limiting behavioral risk, relating to their ability to recognize (i.e., ability to accurately delineate what is doable from what is not) and avoid risky movement behaviors. Military personnel, including pilots and astronauts, must continually integrate sources of perceptual information and make movement judgements that have significant implications in terms of behavioral risk. ${ }^{1,4}$ Further, these judgments often must be made under extreme 
environmental or operational conditions. ${ }^{1,4,23}$ Therefore, perceptual attunement to action boundaries would seem to hold high applicability to these populations.

However, a lack of literature connecting performance-based outcomes to psychological or behavioral health is a critical gap in research on affordances and behavioral risk. Traditional performance measures for alertness or reaction time fail to incorporate any form of perceptualmotor judgement, potentially accounting for this gap in literature. ${ }^{5,15,16}$ While traditional measures require quick responses, they fail to incorporate the critical notions of action boundaries or spatial judgements. Their decisional components are either non-existent (i.e. simply moving finger/mouse when the stimulus is presented) or limited to binary choices (i.e. go / no-go tasks). ${ }^{5,15,16}$

The Perception-Action Coupling Task (PACT) software was designed as an ecologically valid measure of decision-making, with the inclusion of action boundary judgements as the reactionary stimuli. ${ }^{7,9,21}$ Based on the task first described by Smith and Pepping ${ }^{21}$, the PACT is performed on a touchscreen tablet and requires individuals to judge whether a virtual ball can fit inside a virtual hole (aperture). During the assessment, eight ratios of ball-to-aperture size are presented ranging from 0.2 to 1.8 (Fig. 1). The action boundary is represented by a ball-toaperture ratio (B-AR) of 1.0, where the action of moving the ball into the hole is just afforded. At any ratio higher than 1.0, the action would no longer be possible (unafforded). The initial work done to establish the intersession reliability and within-subject stability associated with the PACT is described in detail elsewhere. ${ }^{7}$ The PACT was shown to be reliable and stable over repeated administrations, however it required relatively lengthy familiarization (15 minutes) and data collection (5-10 minutes) periods which may limit usability and induce testing fatigue. ${ }^{7}$ 
Previous literature has demonstrated more consistency in performance of action boundary accuracy assessments when an individual is asked to make judgements with respect to stimuli that are far from a given action boundary. However, as the stimuli approach the action boundary, increased variability and decrements in performance are observed. ${ }^{18,21,22}$ Subsequently, the B-ARs that are furthest from the action boundary on the PACT (i.e. 0.2, 0.4, 1.6, and 1.8) likely have the lowest ability to discern between individuals who perform better (quicker and more accurate) and worse on the task. Further, they may elicit redundant responses with the ratio closest to them (i.e. 0.2 with 0.4 ), given that they present the easiest perceptualjudgements. In other words, on average, participants may perform similarly in response to BARs of 0.4 and 0.2 , even though 0.4 is technically slightly closer to the action boundary.

Given the lengthy time required for familiarization and testing with the PACT the aim of the current study was to explore ways to reduce the necessary testing time of the PACT, thereby increasing its usability. In this effort, two experiments were conducted. Based on the relationship between response times and accuracy and the action boundary described above, the purpose of Experiment 1 was to determine the effects of eliminating B-ARs on the intersession reliability and within-subject stability of the PACT. Data from a previous longitudinal study was recalculated for analysis after iteratively eliminating several B-ARs. We hypothesized that several ratios furthest from the action boundary could be eliminated without affecting the representative behaviors on the PACT described by previous work. ${ }^{7}$ Further, we hypothesized that eliminating these ratios would not change the inherent continuum of reaction times and accuracy of judgements about the action boundary.

\title{
EXPERIMENT 1:
}

\author{
Methods:
}


The complete methods and experimental design for the current study are described in detail elsewhere. ${ }^{7}$ Briefly, 16 subjects $(9$ men, Age $(y r s)=27.8 \pm 3.6)$ were recruited with the inclusion criteria of: ages 18-40, having corrected 20/20 vision, free from any visual impairments, and not taking medications that would impair cognitive processes, alertness or vision. Subjects completed 4 testing sessions, separated by at least 6 days $($ Mean $($ days $)=9.67 \pm 3.4)$. The study protocol was approved in advance by the University of Pittsburgh Institutional Review Board. Each subject provided written informed consent before participating.

Each testing session was identical and consisted of the completion of 9 cycles of the PACT, in a quiet environment with minimal distractions. To perform the PACT (pictured below in Fig. 1), subjects begin with their index or middle finger on the start button. At a randomized interval $(0.34-0.37 \mathrm{sec})$, a virtual ball and aperture appear on the screen. On presentation of the ball-aperture pairing, subjects make their judgement on whether the ball affords posting (can fit) through the aperture, move their finger to the joystick, and either swipe forward if they judge that the ball can fit through the aperture, or backwards if they judge it cannot. Subjects are not given any feedback as to the accuracy of their judgements. Across each cycle of the PACT, each B-AR is presented 16 times, in a randomized order. Each cycle lasts approximately 5 minutes, depending on the speed of responses.

[Fig. 1 Here]

Several variables are calculated from responses to on the PACT. Accuracy of judgements (ACC) is calculated as the percentage of total trials where an accurate judgement was made, with an accurate judgement defined as one where either: the ball afforded posting and the subject moved the joystick forward, or the ball did not afford posting and the subject moved the joystick backward. Reaction time (RT) was determined by the time between presentation of the B-AR 
and the subject making a movement from the start button. Movement time (MT) was determined by the time between the subject making a movement from the start button and initiating a movement with the joystick. Initiation time (IT) was determined by the time between initiation and completion a movement with the joystick. Finally, response time (RST) was determined by the sum of the previous three variables: RT, MT and IT.

As mentioned previously (Sec. 1.0), the study by Connaboy et al. ${ }^{7}$ found that 3 cycles were required for familiarization at the beginning of each session, and 1 to 2 further cycles of testing were necessary to obtain a stable, reliable measure across all variables. Therefore, the first three cycles of testing were eliminated, and all variables were calculated over the $4^{\text {th }}$ cycle ( 1 cycle of testing, 128 trials) and the $4^{\text {th }}$ and $5^{\text {th }}$ cycles combined ( 2 cycles of testing, 256 trials). Finally, variables were initially calculated independently for each B-AR, as opposed to summed across all ratios.

All statistical analyses were performed with IBM SPSS Statistics 23.0 (IBM, Armonk, NY). Descriptive statistics were first calculated for all variables and the means for all variables were plotted by B-AR to examine the response of each to different ratios. Ball-to-aperture ratios were eliminated based on visual inspection of plots, and variables were re-calculated, summed across all remaining ratios, for analysis. Intra-class correlation coefficients [ICC $(3,1)]$ were calculated for all variables for 1 and 2 cycles of testing, to determine the intersession reliability. Coefficients of variation were calculated using the typical error of the measure $\left(\mathrm{CV}_{\mathrm{TE}}\right)$, as described by Hopkins ${ }^{13,14}$. Log transformations were applied to MT before calculating $\mathrm{CV}_{\mathrm{TE}}$, as the variable showed significant departures from normality. ${ }^{13}$ Level for statistical significance was set a priori at: $\alpha=0.05$.

\section{$\underline{\text { Results: }}$}


Visual inspection of plots for the effect of B-AR on PACT variables (Figure 2) showed a typical, quadratic pattern with RT, MT, IT, and RST increasing as B-AR approached 1.0, and ACC decreasing. It also appeared that successive B-ARs furthest from 1.0 (0.2 - 0.4 and $1.6-1.8)$ elicited fairly similar mean responses for most variables (Mean Difference $=1.3 \pm 1.2 \%$ ). Subsequent sets of successive ratios, starting with $0.4-0.6$ and $1.4-1.6$ (Mean Difference $=2.1$ $\pm 2.4 \%$ ), started to show larger differences. Therefore, only the furthest ratios of 0.2 and 1.8 were eliminated. Moving up one B-AR closer to 1.0 (0.4 - 0.6 and 1.4 - 1.6), it did not appear that these successive ratios elicited similar mean responses for most variables, with timing variables beginning to increase slightly and ACC beginning to decrease.

[Fig. 2 Here]

All results for ICCs and $\mathrm{CV}_{\mathrm{TE}}$ are presented in Table 1. Overall, all variables showed good to excellent intersession reliability with both $2(\mathrm{ICC}=0.808-0.985)$ and $1(\mathrm{ICC}=0.709$ 0.937) cycle of testing. Further, all ICCs were statistically significant $(\mathrm{p}<0.05)$. For withinsubject stability, mean $\mathrm{CV}_{\mathrm{TE}}$ ranged from 2.18 - $14.67 \%$ with 2 cycles of testing, and from 3.39 $-19.26 \%$ for 1 cycle of testing.

[Table 1 Here]

\section{EXPERIMENT 2:}

Each cycle of the PACT consists of 16 presentations of each B-AR (Sec. 2.1), in a blockrandomized order, for a total of 128 trials. This large number of trials was selected for the initial version of the PACT, only to ensure that enough trials were completed to capture perceptualmotor decision making during preliminary work. Establishing the minimum number of trials necessary to capture the intended behavioral response from subjects is imperative, given that 
performing more trials than necessary can alter observed response behaviors due to boredom and mental fatigue. Therefore, the purpose of Experiment 2 was to test the effects of eliminating trials on the intersession reliability and within-subject stability of the PACT. Not only would this serve to limit the required testing further, but also to establish the threshold number of trials necessary to obtain adequate reliability and stability across all variables.

A secondary purpose was to establish these same parameters, for all variables, within each individual B-AR. As discussed previously (Sec. 1.0), accuracy and reaction times of affordance judgements are known to be affected by the proximity of the stimulus to the relevant action boundary. ${ }^{18,21,22}$ Further, the results of Experiment 1 confirmed this effect for the PACT, with B-ARs approaching 1.0 eliciting slower, less accurate responses. Subsequently, researchers utilizing the PACT in future studies may be interested in how individuals perform on the PACT in response to certain B-ARs. It may be that environmental, physiological or cognitive stressors will start to elicit changes in performance closer to the action boundary before overall performance begins to deteriorate or will only elicit changes closer to the action boundary. Therefore, we sought to also provide information on the threshold number of trials necessary to obtain adequate reliability and stability for PACT variables for each individual B-AR.

\section{Methods:}

Data collected from the same longitudinal study was utilized for Experiment 2. All PACT variables (RT, MT, IT, RST, ACC) were calculated as described above; averaged across the $4^{\text {th }}$ and $5^{\text {th }}$ cycles of each session, and with the 0.2 and 1.8 B-ARs removed.

Data reduction was iterative, beginning with 32 trials (2 cycles of 16 trials each) of each B-AR, from which 4 trials of each B-AR were eliminated and PACT variables were re-averaged 
across all remaining trials (i.e. $32,28,24, \ldots, 4$ trials). In total, 24 trials were eliminated at each step. This process was utilized in re-calculating all variables averaged across all B-AR, as well as within each B-AR. During the PACT, each B-AR is presented in a block-randomized order, meaning each ratio is presented once before any are presented twice. This allowed for the elimination of an equal number of trials for each B-AR, while still maintaining the sequential order in which trials were presented. In simpler terms, when the first 4 trials were eliminated for each B-AR, the remaining 28 trials were trials $1-28$, in chronological order.

Descriptive statistics were first calculated for all variables. Intra-class correlation coefficients [ICC $(3,1)]$ were calculated for all variables for each iteration of eliminating trials, to determine the intersession reliability. Coefficients of variation were calculated using the typical error of the measure, and a log transformation was applied to all MT coefficients. ${ }^{14}$ Finally, means for all variables were plotted by B-AR, based on the results of these analyses, to reexamine the continuum of responses about the action boundary.

\section{Results:}

Results of ICCs and CV TE with variables averaged across ratios are reported in Table 2. For RT, MT, IT, and RST, ICCs showed good to excellent reliability until the number of remaining trials for each B-AR reached 20 (ICC = 0.817 - 0.973). For ACC, ICCs showed good reliability until the number of remaining trials reached $24(\mathrm{ICC}=0.811)$. In regards to within-subject stability, $\mathrm{CV}_{\mathrm{TE}}$ remained relatively stable for $\mathrm{RT}$ through all trials $\left(\right.$ Mean $\left.\mathrm{CV}_{\mathrm{TE}}=10.89-12.83 \%\right)$ and for ACC through 4 trials $\left(\right.$ Mean $\left.\mathrm{CV}_{\mathrm{TE}}=2.12-3.82 \%\right)$. Initiation time $\left(\right.$ Mean $\mathrm{CV}_{\mathrm{TE}}=10.08-$ $12.05 \%)$ and $\mathrm{RST}\left(\right.$ Mean $\left.\mathrm{CV}_{\mathrm{TE}}=6.57-8.03 \%\right)$ both remained relatively stable through 16 trials. Finally, MT showed the highest $\mathrm{CV}_{\mathrm{TE}}$ and remained stable through 20 trials $\left(\right.$ Mean $\mathrm{CV}_{\mathrm{TE}}=14.67$ $-15.61 \%)$. 
[Table 2 Here]

Results of ICCs and $\mathrm{CV}_{\mathrm{TE}}$ with variables averaged for each individual B-AR are presented in Tables 3 and 4 . To limit the size of tables, results are only reported for averages of 20 trials or greater, given the results for variables averaged across ratios. Across all variables except ACC, all B-ARs showed similar results for intersession reliability with 24 trials of testing, compared to averaging variables across ratios, with ICCs $\geq 0.776$. For ACC, it appears that reliability is reduced when looking at individual B-ARs, with only ratios of $1.4(\mathrm{ICC}=0.726$ $0.797)$ and $1.6(\mathrm{ICC}=0.668-0.720)$ showing similar results compared to averaging ACC across ratios. Coefficients of variation were similar for all B-ARs for IT, RST, and ACC compared to averaging variables across ratios. However, $\mathrm{CV}_{\mathrm{TE}}$ for $\mathrm{RT}$ and $\mathrm{MT}$ showed marked increases for most ratios. Finally, plots depicting means for all variables by B-AR, after removal of ratios and trials (24 trials remaining), are shown in Figure 3.

[Tables 3-4 Here]

[Figure 3 Here]

\section{DISCUSSION:}

The PACT is a novel and ecologically valid assessment of perceptual-motor accuracy and reaction times. ${ }^{7,9,21}$ The purpose of the current study was to explore several methods to reduce PACT testing time, while maintaining the intersession reliability and within-subject stability previously established for this assessment. In this effort, two experiments were undertaken. For Experiment 1, the relationship of reaction times and judgement accuracy on the PACT with BARs was first assessed, and based on these relationships, the ratios of 0.2 and 1.8 were eliminated. Variables were re-calculated, and the effect of removing these two ratios was 
assessed. For Experiment 2, this same process was repeated after eliminating blocks of trials and reliability and within-subject stability coefficients were also calculated for individual B-ARs.

The results of Experiment 1 demonstrated that the ratios furthest from the action boundary could be eliminated from PACT testing without producing a large effect on either intersession reliability or within-subject stability. In the previous study by Connaboy et al. ${ }^{7}$, ICCs for 1 cycle of testing and with all B-ARs included ranged from 0.707 - 0.943. In comparison, IT showed the largest negative effect on reliability with the elimination of ratios, decreasing from 0.906 to 0.899 . Likewise, ICCs for 2 cycles of testing and with all B-ARs were reported to range from 0.820 - 0.992. Again, IT showed the largest negative effect, decreasing from 0.992 to 0.934. More importantly, all variables retained good to excellent reliability with 2 cycles of testing, according to a range of commonly cited criteria for the interpretation of reliability coefficients. ${ }^{2,12,19}$ Further, most variables showed good reliability with only one cycle of testing, however ACC may require 2 (ICC $=0.709$ with 1 cycle) depending on the criteria used. ${ }^{2,12,19}$

Coefficients of variation with all B-ARs were previously reported to range from $2.05-$ $16.03 \%$ with 2 cycles of testing. ${ }^{7}$ Initiation time showed the largest increase in the $\mathrm{CV}_{\mathrm{TE}}$ with elimination of ratios, moving from $4.07 \%$ to $10.71 \%$. For 1 cycle of testing, previously reported $\mathrm{CV}_{\mathrm{TE}}$ ranged from $3.28-19.28 \%$, with IT also showing the largest increase with elimination of ratios, moving from $11.88 \%$ to $12.05 \%$. With only $\mathrm{IT}$ showing a marked increase in $\mathrm{CV}_{\mathrm{TE}}$, and only for 2 cycles of testing, it appears that within-subject stability remained stable with the removal of ratios. In fact, for several variables (MT and $\mathrm{RT}$ ) the $\mathrm{CV}_{\mathrm{TE}}$ were decreased and the ICCs increased, compared to those previously reported, indicating improved stability and reliability. $^{7}$ 
Based on the results of Experiment 1, Experiment 2 moved forward with only B-ARs of 0.4 - 1.6, while retaining 2 cycles of testing. The primary results of Experiment 2 demonstrated that 8 - 12 trials of each B-AR could be eliminated from PACT testing, while still maintaining the intersession reliability and within-subject stability of all variables. All variables maintained good to excellent intersession reliability with the elimination of 12 trials $(\mathrm{ICCs}=0.817-0.973$ ) except for ACC $(\mathrm{ICC}=0.752)$. However, ACC maintained good reliability through the elimination of 8 trials $(\mathrm{ICC}=0.811)$. Within-subject stability results mirrored those for reliability, with $\mathrm{CV}_{\mathrm{TE}}$ remaining relatively unchanged through the elimination of 12 trials for all variables $\left(\mathrm{CV}_{\mathrm{TE}}=2.90-15.40 \%\right)$. With the elimination of further trials, $\mathrm{CV}_{\mathrm{TE}}$ showed a large increase for several variables, including MT (15.40\% to $19.26 \%)$ and IT (10.08\% to $12.05 \%)$.

Finally, PACT variables still show similar or superior intersession reliability and stability, after the elimination of 2 B-ARs and 12 trials for each ratio, compared to other cognitive measures of a similar nature (choice / procedural reaction time measures). Previous literature has reported ICCs ranging from 0.26 - 0.69 for different cognitive performance measures. ${ }^{3,6,20}$ In contrast, the PACT variable with the lowest reliability coefficient still showed an ICC of 0.752 . Comparisons for within-subject stability are more difficult, as the majority of previous studies do not report coefficients of variation. However, 2 studies that have included the standard difference of the error for choice/procedural reaction time tasks, a similar measure of within-subject stability, reported values of $12.77 \%$ and $19.38 \% .{ }^{6,20}$ In comparison, $\mathrm{CV}_{\mathrm{TE}}$ for all PACT variables $(2.90-15.40 \%)$ were within this range or lower.

A secondary purpose of Experiment 2 was to examine ICCs and $\mathrm{CV}_{\mathrm{TE}}$ for PACT variables, averaged for each independent B-AR. This information may be useful for investigators interested in the effects of a given perturbation or intervention on responses to specific B-ARs, or 
on the continuum of responses about the action boundary. ${ }^{18,21,22}$ Overall, the results of these tests showed that reliability was decreased for ACC, and within-subject variability was increased for RT and MT, compared to averaging variables across all B-ARs. While this may be of concern, it is worth noting that ICCs and $\mathrm{CV}_{\mathrm{TE}}$ were comparable to similar cognitive measures. ${ }^{3}$, 6, 20 The addition of further trials (up to 32) resulted in improvements for several variables, within several B-ARs, however most remained unchanged. Finally, plots for all variables by B-AR with 24 trials for each ratio (Fig. 3) showed similar patterns as those before trials were removed (Fig. 2). Initiation time appeared to have a slightly flatter curve, however this variable also showed the flattest pattern about the action boundary before the elimination of trials.

In conclusion, the combined results of Experiment 1 and 2 demonstrate that all PACT variables are reliable and stable when B-ARs are limited to $0.4-1.6$, and only 24 trials of each B-AR are retained. This represents an almost $45 \%$ reduction in total testing time, from 256 trials to 144 . Further, over the 3 -cycle familiarization period, this would result in a $50 \%$ reduction in testing time, from 384 trials to 192. In more practical terms, this means that the shortened PACT would require a familiarization period of approximately 7.5 minutes, and a testing period of 5.5 minutes; down from 15 and 10 minutes respectively. These changes, therefore, would hold great significance in reducing the time-burden of utilizing the PACT, while still maintaining intersession reliability and within-subject stability. When working with pilots, astronauts, or other military personnel, subjects or patients are often under stringent time constraints. Therefore, these reductions in testing burden are highly significant for researchers or clinicians using the PACT to evaluate behavioral risk in these populations.

A limitation of the current study is the extrapolation of previously collected data to these conclusions. While the time sequence for performance of trials was maintained when eliminating 
ratios and trials, the cycles eliminated for familiarization were performed with all trials and ratios. Therefore, we cannot say whether these results would hold if all cycles were performed with the limited ratios and trials outlined above. However, the purpose of this study was to investigate these changes to PACT testing and outline the best course for its future development. In this sense, we believe that these results still hold great significance in providing evidence for the efficacy of a shortened PACT, even if future work is needed to confirm our findings.

ACKNOWLEDGMENTS: This study was funded by the National Aeronautics and Space Administration; grant number NNX15AC13G. The opinions or assertions contained herein are the views of the authors and are not to be constructed as official or reflecting the views of NASA. Further, the funding body did not have a role in the design, analysis, or interpretation of the current study. 


\section{REFERENCES:}

1. Aherne BB, Zhang C, Chen WS, Newman DG. Pilot Decision Making in WeatherRelated Night Fatal Helicopter Emergency Medical Service Accidents. Aerospace medicine and human performance. 2018;89(9):830-6. Epub 2018/08/22. doi: 10.3357/amhp.4991.2018. PubMed PMID: 30126516.

2. Anastasi A. Psychological testing. New York; London: Macmillan; Collier Macmillan; 1988.

3. Ayala F, De Ste Croix M, Sainz de Baranda P, Santonja F. Inter-session reliability and sex-related differences in hamstrings total reaction time, pre-motor time and motor time during eccentric isokinetic contractions in recreational athlete. Journal of electromyography and kinesiology : official journal of the International Society of Electrophysiological Kinesiology. 2014;24(2):200-6. Epub 2014/01/15. doi: 10.1016/j.jelekin.2013.12.006. PubMed PMID: 24418224.

4. Bouak F, Vartanian O, Hofer K, Cheung B. Acute Mild Hypoxic Hypoxia Effects on Cognitive and Simulated Aircraft Pilot Performance. Aerospace medicine and human performance. 2018;89(6):526-35. Epub 2018/05/24. doi: 10.3357/amhp.5022.2018. PubMed PMID: 29789086.

5. Chmura J, Krysztofiak H, Ziemba AW, Nazar K, Kaciuba-Uscilko H. Psychomotor performance during prolonged exercise above and below the blood lactate threshold. Eur J Appl Physiol Occup Physiol. 1998;77(1-2):77-80. Epub 1998/02/12. PubMed PMID: 9459525.

6. Cole WR, Arrieux JP, Schwab K, Ivins BJ, Qashu FM, Lewis SC. Test-retest reliability of four computerized neurocognitive assessment tools in an active duty military population. Archives of Clinical Neuropsychology. 2013;28(7):732-42. 
7. Connaboy C, Johnson CD, LaGoy AD, Pepping G-J, Simpson RJ, Deng Z, et al. Intersession Reliability and Within-Session Stability of a Novel Perception-Action Coupling Task. Aerospace medicine and human performance. 2019;90(2):77-83. doi:

10.3357/AMHP.5190.2019.

8. Daviaux Y, Mignardot J-B, Cornu C, Deschamps T. Effects of total sleep deprivation on the perception of action capabilities. Experimental brain research. 2014;232(7):2243-53.

9. Eagle SR, Nindl BC, Johnson CD, Kontos AP, Connaboy C. Does Concussion Affect Perception-Action Coupling Behavior?: Action Boundary Perception as a Biomarker for Concussion. Clinical Journal of Sports Medicine. 2019;Accepted (in press).

10. Fajen BR, Riley MA, Turvey MT. Information, affordances, and the control of action in sport. International Journal of Sport Psychology. 2009;40(1):79.

11. Gibson James J. The theory of affordances. Perceiving, Acting, and Knowing, Eds Robert Shaw and John Bransford. 1977.

12. Heaton RK, Temkin N, Dikmen S, Avitable N, Taylor MJ, Marcotte TD, et al. Detecting change: A comparison of three neuropsychological methods, using normal and clinical samples. Archives of clinical neuropsychology. 2001;16(1):75-91.

13. Hopkins WG. Measures of reliability in sports medicine and science. Sports medicine. 2000;30(1):1-15.

14. Hopkins WG. Spreadsheets for Analysis of Validity and Reliability. A New View of Statistics [Internet]. 2015; 19:[36-42 pp.].

15. Kopp B, Rist F, Mattler U. N200 in the flanker task as a neurobehavioral tool for investigating executive control. Psychophysiology. 1996;33(3):282-94. 
16. Logan GD, Cowan WB, Davis KA. On the ability to inhibit simple and choice reaction time responses: a model and a method. Journal of Experimental Psychology: Human Perception and Performance. 1984;10(2):276.

17. Palmer CJ, Bigelow C, Van Emmerik RE. Defining soldier equipment trade space: load effects on combat marksmanship and perception-action coupling. Ergonomics. 2013;56(11):1708-21.

18. Pepping G-J, Li F-X. Effects of response task on reaction time and the detection of affordances. Motor Control. 2005;9(2):129-43.

19. Portney L, Watkins M. Foundations of clinical research: application to practice. Stamford, USA: Appleton \& Lange. 1993.

20. Register-Mihalik JK, Guskiewicz KM, Mihalik JP, Schmidt JD, Kerr ZY, McCrea MA. Reliable change, sensitivity, and specificity of a multidimensional concussion assessment battery: implications for caution in clinical practice. The Journal of head trauma rehabilitation. 2013;28(4):274-83.

21. Smith J, Pepping G-J. Effects of affordance perception on the initiation and actualization of action. Ecological Psychology. 2010;22(2):119-49.

22. Stins JF, Michaels CF. Stimulus-response compatibility for absolute and relative spatial correspondence in reaching and in button pressing. The Quarterly Journal of Experimental Psychology: Section A. 2000;53(2):569-89.

23. Vrijkotte S, Roelands B, Meeusen R, Pattyn N. Sustained Military Operations and Cognitive Performance. Aerospace medicine and human performance. 2016;87(8):718-27. Epub 2016/09/17. doi: 10.3357/amhp.4468.2016. PubMed PMID: 27634607. 
24. Withagen R, De Poel HJ, Araújo D, Pepping G-J. Affordances can invite behavior:

Reconsidering the relationship between affordances and agency. New Ideas in Psychology. $2012 ; 30(2): 250-8$. 
Table 1: Intra-class correlation coefficients and coefficients of variation for 2 and 1 cycles of testing and with 0.2 and 1.8 ball-to-aperture ratios removed

\begin{tabular}{lcccc} 
& ICC & $\mathbf{9 5 \%}$ CI & Mean CV $\mathbf{T E}$ & 95\% CI \\
\hline Reaction Time: & & & & \\
2 Cycles & 0.828 & $0.629-0.933$ & 12.83 & $10.42-16.76$ \\
\hline 1 Cycle & 0.824 & $0.623-0.932$ & 11.93 & $9.69-15.59$ \\
\hline Movement Time: & & & & \\
\hline 2 Cycles & 0.985 & $0.968-0.994$ & 14.67 & $11.76-19.59$ \\
\hline 1 Cycle & 0.940 & $0.873-0.977$ & 19.26 & $15.38-25.87$ \\
\hline Initiation Time: & & & & \\
\hline 2 Cycles & 0.934 & $0.844-0.975$ & 10.71 & $8.70-14.00$ \\
\hline 1 Cycle & 0.899 & $0.768-0.962$ & 12.05 & $9.79-15.74$ \\
\hline Response Time: & & & & \\
\hline 2 Cycles & 0.918 & $0.825-0.968$ & 6.61 & $5.37-8.63$ \\
\hline 1 Cycle & 0.870 & $0.722-0.949$ & 8.03 & $6.52-10.49$ \\
\hline Accuracy: & & & & \\
\hline 2 Cycles & 0.808 & $0.595-0.924$ & 2.18 & $1.77-2.84$ \\
\hline 1 Cycle & 0.709 & $0.394-0.885$ & 3.39 & $2.75-4.43$ \\
\hline
\end{tabular}

* ICC $=$ intra-class correlation coefficient, $95 \% \mathrm{CI}=95 \%$ confidence interval, $\mathrm{CV}_{\mathrm{TE}}=$ coefficient of variation using typical error 
Table 2: Intra-class correlation coefficients and coefficients of variation: demonstrating the effects of incrementally removing four trials of each ratio

\begin{tabular}{|c|c|c|c|c|}
\hline & ICC & 95\% CI & Mean $C V_{T E}$ & 95\% CI \\
\hline \multicolumn{5}{|c|}{ Reaction Time: } \\
\hline 32 Trials & 0.828 & $0.629-0.933$ & 12.83 & $10.42-16.76$ \\
\hline 28 Trials & 0.841 & $0.658-0.938$ & 11.08 & $9.00-14.47$ \\
\hline 24 Trials & 0.822 & $0.620-0.931$ & 10.89 & $8.85-14.23$ \\
\hline 20 Trials & 0.817 & $0.608-0.929$ & 11.98 & $9.73-15.65$ \\
\hline 16 Trials & 0.824 & $0.623-0.932$ & 11.93 & $9.69-15.59$ \\
\hline 12 Trials & 0.832 & $0.640-0.935$ & 11.55 & $9.38-15.09$ \\
\hline 8 Trials & 0.828 & $0.629-0.933$ & 12.02 & $9.77-15.71$ \\
\hline 4 Trials & 0.846 & $0.669-0.940$ & 11.55 & $9.38-15.08$ \\
\hline \multicolumn{5}{|c|}{ Movement Time: } \\
\hline 32 Trials & 0.985 & $0.968-0.994$ & 14.67 & $11.76-19.59$ \\
\hline 28 Trials & 0.972 & $0.941-0.989$ & 15.45 & $12.38-20.65$ \\
\hline 24 Trials & 0.974 & $0.945-0.990$ & 15.61 & $12.50-20.86$ \\
\hline 20 Trials & 0.973 & $0.943-0.990$ & 15.40 & $12.34-20.57$ \\
\hline 16 Trials & 0.940 & $0.873-0.977$ & 19.26 & $15.38-25.87$ \\
\hline 12 Trials & 0.919 & $0.828-0.969$ & 23.46 & $18.67-31.70$ \\
\hline 8 Trials & 0.897 & $0.781-0.960$ & 27.88 & $22.11-37.88$ \\
\hline 4 Trials & 0.868 & $0.719-0.948$ & 33.74 & $26.64-46.20$ \\
\hline \multicolumn{5}{|c|}{ Initiation Time: } \\
\hline 32 Trials & 0.934 & $0.844-0.975$ & 10.71 & $8.70-14.00$ \\
\hline 28 Trials & 0.932 & $0.839-0.975$ & 11.29 & $9.17-14.75$ \\
\hline 24 Trials & 0.933 & $0.840-0.975$ & 10.96 & $8.91-14.32$ \\
\hline 20 Trials & 0.935 & $0.841-0.976$ & 10.08 & $8.19-13.17$ \\
\hline 16 Trials & 0.899 & $0.768-0.962$ & 12.05 & $9.79-15.74$ \\
\hline 12 Trials & 0.874 & $0.718-0.951$ & 14.29 & $11.61-18.67$ \\
\hline 8 Trials & 0.853 & $0.673-0.943$ & 16.06 & $13.05-20.98$ \\
\hline 4 Trials & 0.841 & $0.651-0.938$ & 16.91 & $13.74-22.09$ \\
\hline \multicolumn{5}{|c|}{ Response Time: } \\
\hline 32 Trials & 0.918 & $0.825-0.968$ & 6.61 & $5.37-8.63$ \\
\hline 28 Trials & 0.918 & $0.819-0.968$ & 6.63 & $5.38-8.66$ \\
\hline 24 Trials & 0.920 & $0.824-0.969$ & 6.57 & $5.34-8.58$ \\
\hline 20 Trials & 0.918 & $0.818-0.968$ & 7.26 & $5.90-9.49$ \\
\hline 16 Trials & 0.870 & $0.722-0.949$ & 8.03 & $6.52-10.49$ \\
\hline 12 Trials & 0.833 & $0.647-0.934$ & 9.08 & $7.38-11.87$ \\
\hline 8 Trials & 0.815 & $0.610-0.927$ & 9.31 & $7.56-12.17$ \\
\hline 4 Trials & 0.737 & $0.454-0.896$ & 10.85 & $8.81-14.18$ \\
\hline \multicolumn{5}{|l|}{ Accuracy: } \\
\hline 32 Trials & 0.808 & $0.595-0.924$ & 2.18 & $1.77-2.84$ \\
\hline 28 Trials & 0.849 & $0.682-0.941$ & 2.12 & $1.73-2.77$ \\
\hline 24 Trials & 0.811 & $0.595-0.926$ & 2.57 & $2.09-3.36$ \\
\hline 20 Trials & 0.752 & $0.462-0.904$ & 2.90 & $2.35-3.78$ \\
\hline 16 Trials & 0.709 & $0.394-0.885$ & 3.39 & $2.75-4.43$ \\
\hline 12 Trials & 0.685 & $0.344-0.875$ & 3.71 & $3.02-4.85$ \\
\hline 8 Trials & 0.398 & $-0.198-0.755$ & 3.82 & $3.10-4.99$ \\
\hline 4 Trials & 0.336 & $-0.252-0.721$ & 6.33 & $5.14-8.27$ \\
\hline
\end{tabular}


* ICC $=$ intra-class correlation coefficient, $95 \% \mathrm{CI}=95 \%$ confidence interval, $\mathrm{CV}_{\mathrm{TE}}=$ coefficient of variation using typical error 
Table 3: Intra-class correlation coefficients within each ball-to-aperture ratio

\begin{tabular}{lcccccc} 
& $\mathbf{0 . 4}$ & $\mathbf{0 . 6}$ & $\mathbf{0 . 8}$ & $\mathbf{1 . 2}$ & $\mathbf{1 . 4}$ & $\mathbf{1 . 6}$ \\
\hline Reaction Time: & & & & & & \\
32 Trials & 0.872 & 0.824 & 0.793 & 0.693 & 0.815 & 0.830 \\
\hline 28 Trials & 0.879 & 0.827 & 0.807 & 0.713 & 0.833 & 0.837 \\
\hline 24 Trials & 0.861 & 0.807 & 0.776 & 0.685 & 0.814 & 0.831 \\
\hline 20 Trials & 0.831 & 0.779 & 0.671 & 0.588 & 0.752 & 0.813 \\
\hline Movement Time: & & & & & & \\
32 Trials & 0.971 & 0.968 & 0.965 & 0.973 & 0.971 & 0.963 \\
\hline 28 Trials & 0.969 & 0.965 & 0.961 & 0.970 & 0.067 & 0.961 \\
\hline 24 Trials & 0.970 & 0.962 & 0.956 & 0.972 & 0.969 & 0.964 \\
\hline 20 Trials & 0.965 & 0.946 & 0.954 & 0.968 & 0.970 & 0.953 \\
\hline Initiation Time: & & & & & & \\
32 Trials & 0.916 & 0.928 & 0.902 & 0.920 & 0.938 & 0.945 \\
\hline 28 Trials & 0.917 & 0.920 & 0.895 & 0.918 & 0.938 & 0.941 \\
\hline 24 Trials & 0.916 & 0.921 & 0.888 & 0.922 & 0.933 & 0.943 \\
\hline 20 Trials & 0.904 & 0.904 & 0.879 & 0.935 & 0.932 & 0.948 \\
\hline Response Time: & & & & & & \\
32 Trials & 0.930 & 0.905 & 0.856 & 0.880 & 0.910 & 0.912 \\
\hline 28 Trials & 0.930 & 0.901 & 0.861 & 0.859 & 0.894 & 0.914 \\
\hline 24 Trials & 0.931 & 0.892 & 0.833 & 0.850 & 0.896 & 0.921 \\
\hline 20 Trials & 0.910 & 0.855 & 0.844 & 0.850 & 0.865 & 0.889 \\
\hline Accuracy: & 0.590 & 0.621 & 0.657 & 0.547 & 0.811 & 0.666 \\
\hline 32 Trials & 0.565 & 0.638 & 0.681 & 0.615 & 0.822 & 0.711 \\
\hline 28 Trials & 0.423 & 0.607 & 0.696 & 0.578 & 0.797 & 0.668 \\
\hline 24 Trials & 0.435 & 0.494 & 0.544 & 0.357 & 0.726 & 0.720 \\
\hline 20 Trials & & & & & &
\end{tabular}

* All data are results of intra-class correlation coefficients [ICC $(3,1)]$ 
Table 4: Mean coefficients of variation within each ball-to-aperture ratio

\begin{tabular}{lllllll} 
& $\mathbf{0 . 4}(\boldsymbol{\%})$ & $\mathbf{0 . 6}(\boldsymbol{\%})$ & $\mathbf{0 . 8}(\boldsymbol{\%})$ & $\mathbf{1 . 2}(\boldsymbol{\%})$ & $\mathbf{1 . 4}(\boldsymbol{\%})$ & $\mathbf{1 . 6}(\boldsymbol{\%})$ \\
\hline Reaction Time: & & & & & & \\
32 Trials & 12.74 & 14.36 & 13.98 & 14.20 & 13.29 & 12.67 \\
\hline 28 Trials & 12.74 & 13.80 & 14.22 & 14.52 & 13.56 & 13.64 \\
\hline 24 Trials & 12.76 & 14.40 & 15.60 & 15.53 & 13.84 & 13.98 \\
\hline 20 Trials & 15.98 & 15.49 & 17.39 & 17.42 & 14.20 & 14.27 \\
\hline Movement Time: & & & & & & \\
32 Trials & 18.67 & 16.35 & 20.55 & 20.29 & 18.16 & 23.62 \\
\hline 28 Trials & 19.48 & 16.36 & 21.95 & 20.30 & 19.05 & 23.78 \\
\hline 24 Trials & 22.53 & 18.82 & 22.20 & 19.95 & 21.08 & 24.18 \\
\hline 20 Trials & 25.30 & 21.28 & 22.36 & 20.50 & 21.05 & 28.54 \\
\hline Initiation Time: & & & & & & \\
32 Trials & 11.93 & 11.50 & 13.05 & 12.64 & 11.02 & 10.46 \\
\hline 28 Trials & 12.08 & 12.31 & 13.62 & 12.52 & 10.86 & 10.87 \\
\hline 24 Trials & 12.05 & 11.93 & 14.32 & 11.86 & 11.62 & 10.02 \\
\hline 20 Trials & 11.94 & 12.69 & 14.47 & 10.92 & 11.35 & 9.59 \\
\hline Response Time: & & & & & & \\
\hline 32 Trials & 6.81 & 7.19 & 7.76 & 7.57 & 7.26 & 8.41 \\
\hline 28 Trials & 6.96 & 7.21 & 7.73 & 8.09 & 8.21 & 8.73 \\
\hline 24 Trials & 7.40 & 7.84 & 8.11 & 8.45 & 8.32 & 9.08 \\
\hline 20 Trials & 8.95 & 9.21 & 8.66 & 8.56 & 8.52 & 11.25 \\
\hline Accuracy: & 2.94 & 3.11 & 5.46 & 4.37 & 3.60 & 3.36 \\
\hline 32 Trials & 3.22 & 3.40 & 5.36 & 4.51 & 3.45 & 3.80 \\
\hline 28 Trials & 3.80 & 4.46 & 5.74 & 5.54 & 3.87 & 4.48 \\
\hline 24 Trials & 3.10 & 5.19 & 6.84 & 6.43 & 4.61 & 5.26 \\
\hline 20 Trials & & & & & &
\end{tabular}

All data are results of mean coefficients of variation calculated using the typical error of the mean 


\section{FIGURE LEGEND:}

Figure 1: Depiction of PACT interface and example ball-to-aperture ratios

- $\quad$ ball = grey circle at bottom of screen; aperture $=$ white circle at top of screen; start button $=$ circle with fingerprint on bottom right; joystick $=$ circle to left of start button

- Grayscale used for print version; normal PACT interface is colored with yellow ball, white aperture, green start button, and blue joystick.

Figure 2: Means for all PACT variables by ball-to-aperture ratio

Figure 3: Means for all PACT variables by ball-to-aperture ratio: after removal of two ratios and twelve trials from each remaining ratio 